\title{
Efeitos cardiorrespiratórios da buprenorfina em cães anestesiados pelo desfluorano
}

\author{
Cardiorespiratory effects of buprenorphine in dogs anesthetized with desflurane
}

\begin{abstract}
Almir Pereira de Souza ${ }^{1}$ Newton Nunes ${ }^{2}$ Paulo Sérgio Patto dos Santos ${ }^{2}$ Celina Tie Nishimori ${ }^{2}$ Danielli Parrilha de Paula ${ }^{2}$ Piedad Natalia Guerrero ${ }^{2}$ Rosangela Maria Nunes da Silva ${ }^{3}$
\end{abstract}

\section{RESUMO}

Objetivou-se, com este estudo, avaliar os efeitos da buprenorfina sobre variáveis cardiovasculares $e$ respiratórias em cães durante anestesia com desfluorano. Para tanto, foram utilizados 20 cães adultos, distribuídos em dois grupos (GB e GC). A anestesia foi induzida com propofol (8mg $\mathrm{kg}^{-1} \mathrm{IV}$ ) e em seguida os animais foram intubados com sonda de Magill, a qual foi conectada ao aparelho de anestesia para administração de desfluorano (1,5 CAM). Após 30 minutos, foi aplicado no GB buprenorfina $\left(0,02 \mathrm{mg} \mathrm{kg}^{-1}\right) e$ no $\mathrm{GC}$ solução de $\mathrm{NaCl}$ à $0,9 \%\left(0,05 \mathrm{ml} \mathrm{kg}^{-1}\right)$. Avaliaram-se: freqüências cardíaca e respiratória $(F C$ e $f)$; pressões arteriais sistólica, diastólica e média (PAS, PAD e PAM); débito cardíaco (DC); pressão venosa central (PVC); e as variáveis hemogasométricas $\mathrm{pH}, \mathrm{PaCO}_{2}, \mathrm{PaO}_{2}, \mathrm{HCO}_{3}, \mathrm{SatO}_{2}$ e DB. As colheitas dos dados foram feitas aos 30 minutos após o início da administração do desfluorano (MO), 15 minutos após a administração do opióide ou placebo (M15), e a cada 15 minutos após MI5 (M30, M45, M60 e M75). A avaliação estatística dos dados foi efetuada por meio de Análise de Perfil $(P<0,05)$. Houve discreta redução da FC no $G B$, enquanto as outras variáveis cardiovasculares não tiveram redução significativa. A $f$ e o $p H$ tiveram reduções no $G B$, enquanto a $\mathrm{PaCO}_{2}$ esteve aumentada. Concluiu-se que a inclusão da buprenorfina durante anestesia inalatória pelo desfluorano determina discretas alterações cardiovasculares, bem-como potencializa a hipoventilação promovida pelo desfluorano, com a manifestação de hipercapnia, o que não contra-indica o seu uso em pacientes estáveis.

Palavras-chave: opióides, hemogasometria, cães, anestesia.

\begin{abstract}
The aim of this study was to evaluate the effects of buprenorphine on cardiovascular and respiratory variables in dogs anesthetized with desflurane. Twenty adult healthy male and female mongrel dogs were randomly distributed in two groups of ten animals each ( $G B$ and $G C$ ). The anesthetic induction was done using propofol $\left(8 \mathrm{mg} \mathrm{kg}^{-1}, \mathrm{IV}\right)$, and immediately, the dogs were intubated and submited to desflurane anesthesia administrated at 1.5 MAC. After 30 minutes of induction, animals from $G B$ received buprenorphine $\left(0.02 \mathrm{mg} \mathrm{kg}^{-1}\right)$ and $\mathrm{GC}$ saline solution at

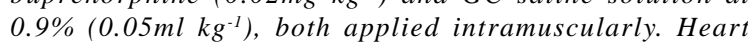
Rate (HR); Systolic, Diastolic and Mean Arterial Blood Pressure (SAP, DAP and MAP); Cardiac Output (CO); Venous Central Pressure (VCP); Respiratory Rate (RR); Corporal Temperature (CT) and hemogasometrics variables. The measurement were realized 30 minutes after beginning the inhalatory anesthesia (MO); 15 minutes after opioid or saline administration (M15). Serial measurements were carried out in 15-minute intervals after M15 (M30, M45, M60 and M75). The numerical data were submited to ANOVA $(P<0.05)$. The $H R, R R$ and $p H$ decreased after opioid administration, while for $\mathrm{PaCO}_{2}$ increased. The results allow us to conclude that buprenorphine determine discreet reduction in the cardiovascular parameters and promotes potentially hypoventilation in dogs anesthetized with desflurane.
\end{abstract}

Key words: opioids, blood gas, dogs, anesthesia.

${ }^{1}$ Departamento de Clínicas Veterinárias, Universidade Federal de Campina Grande (UFCG), Campus de Patos, Av. Universitária, s/ n., 58700-970, Patos, PB, Brasil. E-mail: almir@cstr.ufcg.edu.br. Autor para correspondência.

${ }^{2}$ Departamento de Clínica e Cirurgia Veterinária, Universidade Estadual Paulista (UNESP), Campus de Jaboticabal, SP, Brasil.

${ }^{3}$ Departamento de Medicina Veterinária, UFCG, Campus de Patos, PB, Brasil. 


\section{INTRODUÇÃO}

O desenvolvimento de novos fármacos anestésicos e a elaboração de novas associações representa a possibilidade de acesso a recursos que podem minimizar os riscos inerentes a processos anestésicos rotineiros, com menor comprometimento cardiovascular e, conseqüentemente, redução do risco cirúrgico. Dentre as associações anestésicas existentes e estudadas atualmente, verifica-se a adição de fármacos analgésicos não esteróides, como os opióides. Estes têm se mostrado eficazes em abolir as dores do período pós-operatório, e no trans-anestésico reduzem a quantidade do anestésico geral, seja ele injetável ou inalatório (SOUZA, 2003).

A buprenorfina é um opióide com atividade agonista e antagonista no homem e nos animais (MARTINEZ et al., 1997), com potência analgésica aproximadamente 30 vezes maior que a da morfina (THURMON et al., 1996), aliviando a dor de moderada a grave, associada a procedimentos cirúrgicos abdominais, torácicos e ortopédicos (GÓRNIAK, 2002). E em geral utilizada na dose de 0,005 a $0,02 \mathrm{mg}$ $\mathrm{kg}^{-1}$ pela via intramuscular (FANTONI \& MASTROCINQUE, 2002). Um dos efeitos hemodinâmicos conseqüentes da administração deste opióide, em cães, é a diminuição significativa da freqüência cardíaca, provavelmente por aumento do tono vagal, e alterações discretas na pressão sanguínea sistêmica (STEPIEN et al., 1995). A depressão respiratória, passível de ocorrer em algumas situações, pode ser revertida com o uso de antagonistas opióides, como o naloxone (THURMON et al., 1996). Quanto a via de administração, a buprenorfina pode vir a ser utilizada pelas vias intravenosa (IV) ou intramuscular (IM), pois as variações clínicas observadas são consideradas mínimas (SOUZA et al., 2004).

O desfluorano, um anestésico volátil halogenado fluorinado, que vem sendo utilizado na rotina cirúrgica desde 1992 (WEISKOPF et al., 1992). O baixo coeficiente sangue/gás do desfluorano $(0,42)$, permite rápido aumento ou diminuição da pressão parcial do agente anestésico no sistema nervoso central, o que torna rápidas a indução e a recuperação do paciente (EGER, 1992). No sistema cardiovascular, este anestésico induz queda da pressão arterial média e aumento da freqüência cardíaca (CLARKE et al., 1996), e depressão da contratilidade do coração e conseqüente redução do débito cardíaco (PAGEL et al., 1991). A administração de desfluorano em cães prémedicados com a associação fentanil-droperidol bloqueou a bradicardia produzida por tal associação, e promoveu retardo na condução elétrica atrial, ventricular e átrio-ventricular caracterizada por alterações nas ondas P, QRS e no intervalo PR (SANTOS et al., 2001).

O desfluorano produz, em cães, decréscimo dose-dependente do volume minuto, aumento da freqüência respiratória e depressão da resposta ventilatória ao dióxido de carbono $\left(\mathrm{CO}_{2}\right)$, de maneira diretamente relacionada com a concentração do anestésico, com ou sem a adição de óxido nitroso, registrando-se também, o aumento da pressão parcial arterial de $\mathrm{CO}_{2}\left(\mathrm{PaCO}_{2}\right)$ de acordo com a profundidade do plano anestésico (CLARKE et al., 1996). Desta forma, objetivou-se, com a realização deste estudo, avaliar a manifestação de alterações nas funções cardíacas e respiratórias oriundas da adição do opióide buprenorfina à anestesia inalatória pelo desfluorano, em cães.

\section{MATERIAL E MÉTODOS}

Foram utilizados 20 cães adultos, machos e fêmeas, clinicamente saudáveis, com peso de 11,5kg $( \pm 2,7)$, distribuídos aleatoriamente, ou seja sem distinção de sexo, porte ou peso, em dois grupos de igual número, denominados GC e GB. Após jejum alimentar de 12 horas e restrição hídrica de duas horas, os animais foram induzidos à anestesia com $8 \mathrm{mg} \mathrm{kg}^{-1}$ de propofol ${ }^{\mathrm{a}}$ por via intravenosa (IV) e, em seguida, intubados com sonda de Magill. A sonda foi então coaptada ao aparelho de anestesia volátil para administração do desfluorano ${ }^{\mathrm{b}}$, por meio de circuito anestésico com reinalação parcial de gases ${ }^{c}$, dotado de vaporizador termocompensado, microprocessado e calibrado para o agente anestésico ${ }^{\mathrm{d}}$ tendo como fluxo diluente o oxigênio ( $\left.30 \mathrm{ml} \mathrm{Kg} \mathrm{min}^{-1}\right)$.

O desfluorano foi administrado na concentração de 1,5 CAM, considerando-se a CAM como sendo equivalente a 7,2 V\% (CLARKE et al., 1996), mensurada em equipamento digitale, cujo sensor foi adaptado à extremidade da sonda orotraqueal, conectado ao circuito anestésico.

Após 30 minutos do início da anestesia inalatória, foram administrados, por via intramuscular, buprenorfina $^{\mathrm{f}}\left(0,02 \mathrm{mg} \mathrm{kg}^{-1}\right)$ aos animais do GB e solução de $\mathrm{NaCI}$ à $0,9 \%$ (placebo), no volume de $0,05 \mathrm{ml}$ $\mathrm{kg}^{-1}$ nos animais do grupo GC.

Foram avaliados: freqüência cardíaca (FC), obtida em monitor de eletrocardiografia computadorizado $^{g}$ pelo cálculo do intervalo R-R; pressões arteriais sistólica, média e diastólica (PAS, PAM e PAD, respectivamente), obtidas pela cateterização da artéria femoral esquerda ou direita, cuja leitura foi feita em monitor digital 
computadorizado $^{\text {h}}$; débito cardíaco (DC), obtido pela técnica de termodiluição após introdução do cateter de Swan-Ganz pela veia femoral (esquerda ou direita) até o correto posicionamento na artéria pulmonar; pressão venosa central (PVC) obtida utilizando-se o conduto do cateter de Swan-Ganz utilizado para administrar fluido a baixas temperaturas o qual foi previamente posicionado na veia cava ou átrio, segundo técnica descrita por NUNES (2002); o registro do DC e da PVC foi feito através do monitor digital utilizado para mensurar a pressão arterial; freqüência respiratória $(\mathrm{FR})$, obtida por leitura direta em oxicapnógrafoi; e pH sangüíneo, pressão parcial arterial de dióxido de carbono $\left(\mathrm{PaCO}_{2}\right)$, pressão parcial arterial de oxigênio no sangue arterial $\left(\mathrm{PaO}_{2}\right)$, bicarbonato plasmático $\left(\mathrm{HCO}_{3}\right)$, saturação arterial de oxihemoglobina $\left(\mathrm{SatO}_{2}\right)$ e déficit de base (DB), obtidas da amostra de sangue arterial, colhida da artéria femoral por meio do cateter utilizado para avaliação das pressões arteriais, e analisadas em hemogasômetro digital ${ }^{j}$

O registro das variáveis iniciou-se imediatamente antes da aplicação do opióide ou placebo (MO). Novas mensurações foram feitas 15 minutos após a aplicação dos fármacos (M 15) e de 15 em 15 minutos após M15 (M30, M45, M60 e M75). A análise estatística das variáveis foi efetuada por meio de Análise de Perfil (MORRISON, 1967; CURI, 1980), considerando um nível designificância de 5\% $(p<0,05)$.

\section{RESULTADOS E DISCUSSÃO}

Observou-se na FC dos animais deste experimento discretas variações, mas mantendo as médias, de uma forma geral, dentro de parâmetros considerados normais para a espécie (Tabela l). Os animais tratados com buprenorfina apresentaram redução dos seus valores quando comparados ao momento inicial das mensurações mas que não diferenciaram do GC. Estas reduções, apesar de discretas, estariam diretamente relacionadas à ação vagal observada quando do uso desta classe de agentes (MARTINEZ et al., 1997; LUCAS et al., 2001). No grupo de animais em que foi administrado unicamente desfluorano as médias de FC permaneceram estáveis, dentro dos valores fisiológicos para a espécie, não sendo possível identificar acréscimo da atividade simpatomimética característica quando do uso deste fármaco (WEISKOPF et al., 1990; ZWASS et al., 1992; PICKER et al., 2001).

Na avaliação da pressão arterial (PA), foi possível constatar que a buprenorfina não interferiu nos resultados, permanecendo o parâmetro estável ao longo do período experimental. Este achado permite concordar com os relatos encontrados na literatura que ressaltam os discretos efeitos depressores cardiovasculares desse agente (STEPIEN et al., 1995). Entretanto, é válido conjecturar que o desfluorano, associado à buprenorfina, possa ter contribuído para a melhor estabilidade circulatória, pois quando da utilização de isofluorano ou halotano, MARTINEZ et al. (1997) anotaram redução significativa da PA a qual estaria relacionado a diminuição do índice cardíaco secundariamente ao decréscimo da FC para o isofluorano ou pela redução da FC e do volume sistólico durante anestesia com halotano.

Com relação ao débito cardíaco (DC), não foi registrada diferença significativa entre os animais do GB quando comparados ao grupo controle, concordando com os achados da literatura (GREENE et al., 1990; STEPIEN et al., 1995). As principais manifestações de alterações cardiovasculares observadas para os opióides estão associadas à via de administração utilizada, pesando o fato da via IV causar alterações mais evidentes como a bradicardia (COPLAND et al., 1987; GROSS et al., 1993; LUCAS et al., 2001). MARTINEZ et al. (1997), utilizaram a via IV para administrar a buprenorfina e registraram redução nas variáveis cardiovasculares, entre elas do DC e do índice cardíaco. Entretanto, tais alterações não foram observadas de forma significativas por SOUZA et al. (2004).

Em relação à PVC, os animais do grupo GC apresentaram médias que decresceram durante o estudo. Entretanto, este resultado não foi considerado significativo pelo teste estatístico empregado (Tabela 1). Tal achado está em desacordo com aquele encontrado por CLARKE et al. (1996) que obtiveram elevação do parâmetro a qual estaria relacionada à depressão do miocárdio com efeito inotrópico negativo, o que pode ter acontecido em função dos autores terem empregado diferentes concentrações anestésicas, com as doses mais elevadas em tese afetando mais intensamente a contratilidade do miocárdio. No grupo GB, as médias da PVC estabilizaram-se ao longo do período experimental. Este achado pode estar relacionado à manutenção da contratilidade cardíaca associada à diminuição da resistência periférica, que desta forma reduziria a póscarga permitindo a manutenção do retomo venoso. No que tange ao parâmetro FR, as variações observadas no grupo tratado com buprenorfina foi discreta, não diferindo daquela observada no grupo placebo. De fato, os opióides agonistas- antagonistas proporcionam menor depressão respiratória devido a 
Tabela 1 - Valores médios e desvio padrão, entre parênteses, das variáveis clínicas freqüência cardíaca (FC), freqüência respiratória (f), pressões arteriais sistólic, diastólica e média (PAS, PAD e PAM, respectivamente), débito cardíaco (DC) e pressão venosa central (PVC), em cães tratados com placebo (GC) ou buprenorfina (GB) durante anestesia gera com desfluorano, em diferentes momentos.

\begin{tabular}{|c|c|c|c|c|c|c|c|}
\hline Var. & Grupo & M0 & M15 & M30 & M45 & M60 & M75 \\
\hline$\overline{\text { FC }}$ & GC & $122(11,5)$ & $124(13,9)$ & $124(15,1)$ & $123(18,8)$ & $120(14,6)$ & $119(13,8)$ \\
\hline (bat./min.) & GB & 131(17,9)a & $116(24,9)$ & $112(24,2) b$ & $111(17,7)$ & $111(16,7)$ & $113(14,1)$ \\
\hline f & GC & $15(6,6)$ & $18(8,8)$ & $18(8,4)$ & $18(8,5)$ & $16(6,5)$ & $19(9,0)$ \\
\hline (mov./min.) & GB & $15(7,5)$ & $14(8,5)$ & $12(6,8)$ & $12(6,7)$ & $11(6,0)$ & $12(4,6)$ \\
\hline PAS & GC & $112(8,5)$ & $114(12,5)$ & $116(14,2)$ & $115(14,0)$ & $113(11,3)$ & $114(12,3)$ \\
\hline (mmHg) & GB & $103(21,0)$ & $102(19,1)$ & $105(15,5)$ & $102(14,4)$ & $103(13,5)$ & $102(11,4)$ \\
\hline $\mathrm{PAD}$ & GC & $64(10,9)$ & $64(12,7)$ & $66(14,8)$ & $65(14,7) \mathrm{A}$ & $62(11,2)$ & $61(9,6)$ \\
\hline (mmHg) & GB & $58(13,7)$ & $54(14,9)$ & $53(11,2)$ & 52(9,3)B & $52(8,8)$ & $54(9,3)$ \\
\hline PAM & GC & $82(10,3)$ & $83(12,8)$ & $86(15,4)$ & $85(15,8)$ & $81(12,0)$ & $82(13,8)$ \\
\hline (mmHg) & GB & $77(16,7)$ & $74(17,1)$ & $75(12,7)$ & $73(11,2)$ & $73(10,6)$ & $74(9,7)$ \\
\hline DC & GC & $2,28(0,47)$ & $2,45(0,75)$ & $2,58(0,85)$ & $2,53(0,78)$ & $2,42(0,68)$ & $2,51(0,72)$ \\
\hline (litros./min.) & GB & $2,43(0,72)$ & $2,37(0,34)$ & $2,28(0,60)$ & $2,44(0,75)$ & $2,37(0,70)$ & $2,43(0,69)$ \\
\hline PVC & GC & $2,0(2,1)$ & $1,4(2,2)$ & $1,7(2,2)$ & $1,1(2,5)$ & $1,2(2,0)$ & $1,1(2,2)$ \\
\hline (mmHg) & GB & $2,1(2,2)$ & $2,1(2,5)$ & $2,3(2,2)$ & $2,5(1,9)$ & $2,3(1,8)$ & $2,4(1,7)$ \\
\hline
\end{tabular}

Médias seguidas da mesma letra não apresentam diferenças estatisticamente significativas entre si pela Análise de Perfil (P<0,05). Letras maiúsculas representam a diferença entre grupos. Letras minúsculas representam a diferença entre os momentos dentro cada grupo.

sua maior seletividade por receptores kappa (HOSGOOD, 1990; JAFFE \& MARTIN, 1991), não tão presentes nos centros bulbares. No GC, a freqüência respiratória permaneceu estável durante todo o período experimental. Isto deveu-se principalmente ao uso de CAM fixa, pois a depressão respiratória produzida pelo desfluorano é dependente da dose (WARLTIER \& PAGEL, 1992; STEFFEY, 1996; SANTOS et ai., 2001; NISHIMORI, 2003).

$\mathrm{O}$ pH é determinado pela concentração de íons $\mathrm{H}^{+}$no sangue que por sua vez terá sua quantidade aumentada à medida que a concentração de dióxido de carbono no sangue decresce (O’FLAHERTY, 1994). Neste estudo, foi possível constatar que em ambos os grupos as médias do $\mathrm{pH}$ estiveram sempre abaixo de 7,35 que seria o mínimo necessário para o funcionamento normal dos processos celulares (CUNNINGHAM, 2004). Entretanto, a adição do opióide determinou, 30 minutos após sua administração, decréscimo destes valores que se tomaram inferiores àqueles registrados nos animais do grupo controle (GB - 7,22; GC - 7,31), sendo tais achados compatíveis com a redução da FR, observada no GB (Tabela l).

Analisando-se os valores obtidos para a $\mathrm{PaCO}_{2}$, é possível observar que a média do grupo GB esteve acima do limite recomendável de $45 \mathrm{mmHg}$, para pacientes anestesiados recebendo $100 \%$ de $\mathrm{O}_{2}$ (MUIR III; HUBBELL, 1997). Tal achado demonstra a capacidade da buprenorfina em determinar hipercapnia em pacientes anestesiados pelo desfluorano. Entretanto, estes valores mantiveram-se relativamente estáveis, a despeito da discreta redução da FR, o que indica que a ventilação alveolar foi pouco alterada.

O quadro de hipercapnia observado nos animais que receberam buprenorfina deveu-se à uma discreta hipoventilação e não à hipoxemia, com diminuição do aporte de oxigênio aos tecidos, visto que os valores de $\mathrm{Pa}_{2}$ (Tabela 2 ) mantiveram-se sempre acima dos limites necessários de $\mathrm{O}_{2}$, ou seja $110 \mathrm{mmHg}$ (MUIR III \& HUBBELL, 1997), o qual desta forma fez permanecer a saturação de oxihemoglobina em 100\%. Em relação à $\mathrm{SatO}_{2}$, não foi registrada em quaisquer dos grupos variações significativas, mantendo as médias estáveis e dentro da faixa de normalidade para cães recebendo oxigênio à 100\% (HASKINS, 1996). Embora esta variável por si só não represente a quantidade de oxigênio disponível para os tecidos (NICHOLSON, 1996), esta permite determinar a oxigenação arterial (JONES, 1996) e serve como parâmetro de monitoramento de distúrbios que possam pôr o paciente em risco, durante procedimentos anestésicos.

O estudo da variável bicarbonato plasmático $\left(\mathrm{HCO}_{3}^{-}\right.$permite avaliar a resposta do organismo frente a variações do $\mathrm{pH}$ no sangue. Segundo LUNA (2002), o bicarbonato é responsável por mais de $50 \%$ da capacidade tampão extracelular, podendo variar de 18 a $24 \mathrm{mEq} \mathrm{L}{ }^{-1}$. Neste estudo, verificou-se que, o grupo tratado com buprenorfina as 
Tabela 2 - Valores médios e desvio padrão, entre parênteses, do $\mathrm{pH}$ sangüíneo $(\mathrm{pH})$, pressão parcial arterial de dióxido de carbono (PaCO ${ }_{2}$ ), pressão parcial arterial de oxigênio no sangue arterial $\left(\mathrm{PaO}_{2}\right)$, bicarbonato plasmático $\left(\mathrm{HCO}_{3}\right)$, saturação arterial de oxihemoglobina $\left(\mathrm{SatO}_{2}\right)$ e déficit de base (DB), em cães tratados com placebo (GC) ou buprenorfina (GB) durante anestesia geral com desfluorano, em diferentes momentos.

\begin{tabular}{|c|c|c|c|c|c|c|c|}
\hline Var. & Grupo & M0 & M15 & M30 & M45 & M60 & M75 \\
\hline \multirow{2}{*}{$\mathrm{pH}$} & GC & $7,29(5,9)$ & $7,31(5,6)$ & 7,31(3,8)A & 7,32(4,3)A & $7,30(4,7) \mathrm{A}$ & $7,29(4,7) \mathrm{A}$ \\
\hline & GB & 7,26(8,1)a & $7,24(8,0)$ & $7,22(7,8) \mathrm{B}$ & 7,21(8,1)B & 7,20(7,3)B & 7,20(8,1)Bb \\
\hline \multirow{2}{*}{$\begin{array}{l}\mathrm{PaCO}_{2} \\
(\mathrm{mmHg})\end{array}$} & GC & $44,8(7,7)$ & 43,8(6,9)A & $43,1(5,2)$ & $41,8(5,0)$ & $43,7(6,6) \mathrm{A}$ & 43,8(6,6)A \\
\hline & GB & $52,5(9,5) a$ & 55,1(11,1)B & $57,6(11,6)$ & $60,6(12,1)$ & $61,1(13,0) \mathrm{B}$ & $62,2(12,8) \mathrm{Bb}$ \\
\hline \multirow{2}{*}{$\begin{array}{l}\mathrm{PaO}_{2} \\
(\mathrm{mmHg})\end{array}$} & GC & $435(52,8)$ & $436(52,0)$ & $450(57,0)$ & $425(57,0)$ & $447(59,8)$ & $450(63,0)$ \\
\hline & GB & $417(56,7)$ & $435(78,8)$ & $449(83,1)$ & $446(49,1)$ & $457(48,7)$ & $456(57,6)$ \\
\hline \multirow{2}{*}{$\begin{array}{l}\mathrm{SatO}_{2} \\
(\%)\end{array}$} & GC & $100(0)$ & $100(0)$ & $100(0)$ & $100(0)$ & $100(0)$ & $100(0)$ \\
\hline & GB & $100(0)$ & $100(0)$ & $100(0)$ & $100(0)$ & $100(0)$ & $100(0)$ \\
\hline \multirow{2}{*}{$\mathrm{HCO}_{3}$} & GC & $22(2,6)$ & $22(2,8)$ & $22(3,0)$ & $22(2,9)$ & $22(3,5)$ & $22(2,9)$ \\
\hline & GB & $24(2,1)$ & $24(2,9)$ & $24(3,0)$ & $24(2,6)$ & $24(3,2)$ & $24(2,7)$ \\
\hline \multirow{2}{*}{ DB } & GC & $-4,4(3,2)$ & $-4,1(3,0)$ & $-4,1(3,5)$ & $-4,8(3,2)$ & $-4,5(3,7)$ & $-4,2(3,4)$ \\
\hline & GB & $-3,1(2,6)$ & $-3,3(3,0)$ & $-3,8(3,7)$ & $-3,8(2,9)$ & $-3,8(3,1)$ & $-3,6(2,6)$ \\
\hline
\end{tabular}

Médias seguidas da mesma letra não apresentam diferenças estatisticamente significativas entre si pela Análise de Perfil (P<0,05). Letras maiúsculas representam a diferença entre grupos. Letras minúsculas representam a diferença entre os momentos dentro de cada grupo.

médias do $\mathrm{HCO}_{3}{ }^{-}$estiveram sempre próximas do limite máximo recomendável. Tal achado está diretamente correlacionado às elevações observadas na $\mathrm{PaCO}_{2}$, pois o mecanismo compensatório ativado pelo organismo aumenta de $\mathrm{l}$ a $2 \mathrm{mEq} \mathrm{L}^{-1}$ de $\mathrm{HCO}_{3}{ }^{-}$para cada aumento agudo de $10 \mathrm{mmHg}$ de $\mathrm{PaCO}_{2}$ (MUIR III \& HUBBELL, 1997) a fim de neutralizar o excesso de $\mathrm{CO}_{2}$ presente no sangue.

As médias do DB permaneceram dentro dos parâmetros considerados normais, ou seja, 5 a $-5 \mathrm{MEq}$ $\mathrm{L}^{-1}$ (MUIR III; HUBBELL, 1997). Tais resultados, associados à permanência das médias de $\mathrm{HCO}_{3}{ }^{-}$em parâmetros normais permite descaracterizar quadro de acidose metabólica. Desta forma, o decréscimo de $\mathrm{pH}$ registrado aliado à elevação da $\mathrm{PaCO}_{2}$ são realmente compatíveis com acidose respiratória, em virtude da redução da FR (LUCAS et al., 2001; LUNA, 2002).

\section{CONCLUSÃO}

Os resultados obtidos com a metodologia proposta permite concluir que a buprenorfina determina discreta alterações cardiovasculares, que não contra-indicam seu uso em pacientes hemodinamicamente estáveis. Entretanto, o uso deste opióide determina hipercapnia em cães anestesiados com desfluorano devido a potencialização da hipoventilação promovida pelo anestésico geral.

\section{AGRADECIMENTOS}

Os autores agradecem à Fundação de Amparo à Pesquisa do Estado de São Paulo (FAPESP), pelo apoio financeiro concedido.

\section{FONTESDEAQUISIÇÃO}

a - DIPRIVAN - Zeneca Farmacêutica do Brasil Ltda. - São Paulo, SP, Brasil.

b - SUPRANE - Zeneca Farmacêutica do Brasil Ltda. - São Paulo, SP, Brasil.

c - OHMEDA - mod. Excel 210SE - Datex Ohmeda - Miami, EUA.

d - OHMEDA - mod. TEC 6 - Datex Ohmeda - Miami, EUA. e - OHMEDA - mod. RGM 5220 - Datex Ohmeda - Miami, EUA.

f- TEMGESIC - Ind. Quim. e Farm. Schering-plough S/A, São Paulo, SP, Brasil.

g - TEB - mod. ECGPC software versão 1.10 - São Paulo.SP, Brasil.

h - DIXTAL 2010 - Dixtal do Brasil, Manaus, AM, Brasil.

i - OXICAPNÓGRAFO DX 7100 - Dixtal do Brasil, Manaus, AM, Brasil.

j - I-STAT-Sensor Services, Inc. — New Jersey, EUA.

\section{REFERÊNCIAS}

CLARKE, K.W. et al. Cardiopulmonary effects of desflurane in the dog during spontaneous and artificial ventilation. Res Vet Sc, v.61, p.82-86, 1996.

COPLAND, V.S. et al. Oxymorphone: cardiovascular, pulmonary, and behavioral effects in dogs. Am J.Vet Res. v.48, n.l l, p.1626-1630, 1987.

CUNNINGHAM, J.G. Tratado de fisiologia veterinária. 3.ed. Rio de Janeiro: Guanabara Koogan, 2004. 577p.

CURI, P.R. Análise de medidas repetidas em experimentos biológicos. Rev Bras Estat, v.41, p.137-150, 1980.

EGER, E.I II. Desflurane animal and human pharmacology: aspects of kinetics, safety, and MAC. Anesth Analg, v.75, p.3-9, 1992.

FANTONI, D.T.; MASTROCINQUE, S. Fisiopatologia e controle da dor. In: FANTONI, D.T.; CORTOPASSI, S.R.G. 
Anestesia em cães e gatos. São Paulo: Roca, 2002. Cap.31, p.323-336.

GÓRNIAK, S.L. Hipnoanalgésicos e neuroleptoanalgesia. In: SPINOSA, H.S. et al. (Eds). Farmacologia aplicada à medicina veterinária. 3.ed. Rio de Janeiro: Guanabara Koogan, 2002. Cap.15, p.158-165.

GREENE, S.A. et al. Cardiovascular effects of butorphanol in halothane-anesthetized dogs. Am J Vet Res, v.51, n.8, p.1276-1279, 1990.

GROSS, M.E. et al. Cardiorespiratory effects of combined midazolam and butorphanol in isoflurane-anesthetized cats. Vet Surg, v.22, n.2, p.159-162, 1993.

HASKINS, S.C. Monitoring the anesthetized patient. In: THURMON, J.C. Lumb \& Jones' veterinary anesthesia. 3.ed. Philadelphia: Lea \& Feabiger, 1996. p.409-424.

HOSGOOD, G. Pharmacologic features of butorphanol in dogs and cats. J Am Vet med Assoc, v.196, p.135-136, 1990.

JAFFE, J.H.; MARTIN, W.R. Analgésicos opióides e antagonistas. In: GILMAN, A.G et al. (Eds). Goodman \& Gilman - As bases farmacológicas da terapêutica. 8.ed. Rio de Janeiro: Guanabara 1991. p.320-343.

JONES, J.L. Noninvasive monitoring techniques in anesthetized animais. Vet Med, v.4, p.326335,1996.

LUCAS, A.N. et al. Comparison of the effects of morphine administered by constant-rate intravenous infusion or intermittent intramuscular injection in dogs. J Am Vet Med Assoc, v.218, n.8, p.884-891, 2001.

LUNA, S.P.L. Equilíbrio acido-básico. In: FANTONI, D.T.; CORTOPASSI, S.R.G. Anestesia em cães e gatos. São Paulo: Rocca, 2002. Cap.10, p.120-129.

MARTINEZ, E.A. et al. Cardiovascular effects of buprenorphine in anesthetized dogs. Am J Vet Res, v.58, n.11, p.1280-1283, 1997.

MORRISON, D.F. Multivariate statistical methods. New York: McGrows Hill, 1967. 388p.

MUIR III, W.W.; HUBBELL, J.A.E. Manual de anestesia veterinária. 2.ed. Madrid: Mosby/DoymaLibros, 1997. 503p.

NICHOLSON, A. Monitoring techniques and equipment for small animal anaesthesia. Austr Vet J, v.74, n.2, p.114-123, 1996.

NISHIMORI, C.T. Efeitos do óxido nitroso sobre parâmetros cardiovasculares, respiratórios e concentração alveolar mínima do desfluorano, em cães. 2003. 87f. Dissertação (Mestrado em Cirurgia Veterinária) - Faculdade de Ciências Agrárias e Veterinárias, Universidade Estadual Paulista, Jaboticabal.
NUNES, N. Monitoração da anestesia. In: FANTONI, D.T.; CORTOPASSI, S.R.G. Anestesia em cães e gatos. São Paulo: Rocca, 2002. Cap.6, p.64-81.

O'FLAHERTY, D. Carbon dioxide and monitoring. In: Capnography - Principles and practice series. London: BMJ Publishing Group, 1994. p.3-20.

PAGEL, P.S. et al. Influence of volatile anesthetics on myocardial contractily in vivo: desflurane versus isoflurane. Anesthesiology, v.74, p.900- 907, 1991.

PICKER, O. et al. Inhalation anaesthetics increase heart rate by decreasing cardiac vagal activity in dogs. Br J Anaesth, v.87, n.5, p.748-754, 2001.

SANTOS, P.S.P. et al. Eletrocardiografia de cães submetidos a diferentes concentrações de desflurano, pré-tratados ou não com a associação de fentanil-droperidol. Ciência Rural, v.31, n.5, p.805-811, 2001.

SOUZA, A.P. Efeitos cardiovasculares, hemogasométricos e neuroendócrinos da buprenorfína, butorfanol ou morfina, em cães anestesiados pelo desflurano. 2003. 104f. Tese (Doutorado em Cirurgia Veterinária) - Faculdade de Ciências Agrárias e Veterinárias, Universidade Estadual Paulista, Jaboticabal.

SOUZA, A.P. et al. Avaliação da buprenorfina pelas vias intravenosa ou intramuscular em cães anestesiados pelo desflurano. Ciência Rural, v.34, n.3, p.809-814, 2004.

STEFFEY, E.P. Inhalation anesthetics. In: THURMON, J.C. et al. (Eds). Lumb \& Jones' veterinary anesthesia. 3.ed. Philadelphia: Lea \& Feabiger, 1996. p.297-329.

STEPIEN, R.L. et al. Cardiorespiratory effects of acepromazine maleate and buprenorphine hydrochloride in clinically normal dogs. Am J Vet Res, v.56, n.l, p.78-84, 1995.

THURMON, J.C. et al. Injectable anesthetics. In: Lumb \& Jones' veterinary anesthesia. 3.ed. Philadelphia: Lea \& Feabiger, 1996. Chapt.9, p.210-240.

WARLTIER, D.C.; PAGEL, P.S. Cardiovascular and respiratory actions of desflurane: is desflurane different from isoflurane? Anesth Analg, v.75, p.17-31, 1992.

WEISKOPF, R.B. et al. Cardiovascular actions of common anesthetic adjuvants during desflurane (I-653) and isoflurane anesthesia in swine. Anesth Analg, v.71, n.2, p.144-148, 1990.

WEISKOPF, R.B. et al. Desflurane does not produce hepatic or renal injury in human volunteers. Anesth Analg, v.74, p.570-574, 1992.

ZWASS, M.S. et al. Induction and maintenance characteristics of anesthesia with desflurane and nitrous oxide in infants and children. Anesthesiology, Iowa, v.76, n.3, p.373-378, 1992. 